\title{
Comunicación

\section{Persona multicultural, comunicación intercultural. La propuesta de Amin Maalouf}

\author{
MARÍA TERESA NICOLÁS GAVILÁN 1
}

Amín Maalouf propone un modo de concebir la identidad personal en la época de las tribus planetarias: la identidad compuesta o multicultural. El escritor libanés señala los riesgos que se corren si se rechazan estas identidades multiculturales, que pueden convertirse en identidades asesinas. Entender a la persona multicultural permitirá una correcta comunicación intercultural en Latinoamérica.

PALABRAS CLAVES: identidad, globalización, cultura, integración, violencia.
Amin Maalouf proposes a way of conceiving personal identity in the era of planetary tribes: the composite or multicultural identity. The Lebanese writer points out the risks involved if one rejects these multicultural identities, that can become murderous. Understanding the multicultural person will correct intercultural communication in Latin America.

KEY WORDS: identity, globalization, culture, integration, violence.

1 Universidad Panamericana, México.

Correo electrónico: mnicolas@up.edu.mx 


\section{INTRODUCCIÓN}

Se puede considerar que todas las épocas han tenido sus factores de grandes transformaciones sociales, políticas y culturales. Hoy en día este motor tiene un nombre: multiculturalismo.

Los problemas que se derivan de la realidad multicultural pueden ser analizados desde muchas perspectivas, agrupándose éstas en dos grandes visiones: la social -la dimensión política, la sociológica, la jurídica- y la personal -la antropológica y la pedagógica.

Hasta ahora la mayoría de estudios y aportaciones que abordan el tema multicultural lo hacen desde la perspectiva social (Appadurai, 2002; Bauman, 2001, 1999; García-Canclini, 1999; Giddens, 2000), también los medios de comunicación de distintos países establecen (agenda setting) los siguientes temas de análisis y debate: el problema de los inmigrantes y los países de acogida; la integración de los indígenas en las comunidades modernas, y la viabilidad de Estados integrados por diversas comunidades nacionales.

Sin embargo, la persona es la base de la sociedad y, por lo tanto, para entender a una sociedad multicultural, hay que empezar por estudiar y comprender a la persona multicultural, y concretamente la identidad, que este concepto, como señaló Giménez (2000, 2005), sigue siendo una noción imprescindible en las ciencias sociales, que ha venido a potenciar la teoría de la acción social y, particularmente la acción comunicativa.

A partir de lo antes mencionado, considero que la pregunta clave es la que propone Castells (1997): “ ¿se puede remediar la tendencia opuesta en todo el mundo, la distancia creciente entre globalización e identidad, entre la red y el yo?" (prólogo).

Considero que el autor libanés Amin Maalouf ofrece una interesante propuesta sobre esta cuestión. Su elección parece razonable y atractiva; razonable porque él encarna en sí mismo su proposición: la identidad compuesta, y atractiva por el modo en que están escritas sus novelas y su ensayo, y por el contenido de las mismas.

Maalouf nació el 25 de febrero de 1949, en Beirut, Líbano, país en el que convivían pacíficamente más de una quincena de comunidades culturales. Su familia es de origen árabe, de religión católica -rito malaquita- y con una larga tradición cultural. Es nieto e hijo de poeta; su padre fundó dos diarios en Beirut. 
Cursó los estudios de francés con los jesuitas e ingresó en la Facultad de Sociología. A los 22 años, comenzaba a ejercer como articulista para un periódico libanés, llegando a corresponsal de guerra en el extranjero, lo que le dio la oportunidad de viajar y conocer otras regiones: Bangladesh, Vietnam, Etiopía, América del Sur, etcétera.

En 1975 su país entró en guerra civil y se marchó a París con su familia. Ejerció como periodista llegando a ser redactor jefe del semanal Jeune Afrique. Posteriormente decidió empezar a escribir. En 1983 apareció su primer libro Las cruzadas vistas por los árabes; sus siguientes obras fueron: León el Africano, Los jardines de la luz, Samarcanda, etc. En 1993 ganó el prestigioso Premio Goncourt, el más importante galardón de la literatura francesa, con la novela La roca de Tanios.

Él ha escrito en diversos géneros: la novela, el ensayo y su último trabajo -hasta la fecha- ha sido el libreto para la ópera El amor de lejos, publicada en edición bilingüe (castellano y francés) en 2002.

Como afirma Maalouf en su propia biografía se encuentran entrelazadas dos culturas.

Las fuentes para el análisis del pensamiento de Maalouf pertenecen a distintos géneros: el ensayo, la novela y la entrevista. Se han acotado a las siguientes obras: Identidades asesinas (ensayo), León el Africano, La roca de Tanios, Las escalas de Levante y El viaje de Baldassare (novelas), y varias entrevistas realizadas y publicadas tanto en prensa como en Internet. Las ediciones de las obras literarias antes mencionadas son las publicadas por Editorial Alianza; se ha optado por esta versión en castellano y no por su original en francés, ya que el estudio realizado no versa sobre su estilo literario u otras cuestiones que harían necesario trabajar con el texto original.

Es importante advertir lo delicado que es abordar este tema de la identidad, ya que el debate sobre la persona, como apunta Toscano (2001) "no se presta a compromisos y mediaciones, ... perder no es perder algo, sino a sí mismo" (p. 30 ).

\section{LA IDENTIDAD PERSONAL Y LA GLOBALIZACIÓN}

Entre las definiciones de identidad que se han consultado se ha elegido la que propone Giménez (2000), ya que se considera muy completa y acorde con los propósitos de este artículo. El autor dice que identidad es: 
El conjunto de repertorios culturales interiorizados (representaciones, valores, símbolos ...) a través de los cuáles los actores sociales (individuos o colectividades) demarcan simbólicamente sus fronteras y se distinguen de los demás actores sociales en una situación determinada, todo ello en contextos históricamente específicos y socialmente estructurados (p. 2).

Se puede decir que la identidad es la cultura propia de un grupo social interiorizada de una manera particular por cada persona. Las personas comparten una cultura, pero la interiorizan o asimilan de manera particular. La identidad es la cultura interiorizada por la persona según sus propias circunstancias: de tiempo y espacio. Es aquello que hace que un individuo se reconozca, distinga y proyecte.

La pregunta es, ¿puede haber una identidad global? Entre los teóricos de la globalización no faltan los que afirman, no sólo la existencia, sino también la intensificación y la ampliación creciente de un sentido de pertenencia global que implicaría la percepción del mundo como una comunidad globalizada. Según diversos autores la conciencia global del mundo como un todo, alimentada por experiencias inducidas a través de los medios de comunicación y estimulada por las primeras fotografías de la tierra desde el espacio, habría alcanzado un nivel de masa a partir de los años setenta (Featherstone, 1992).

En este sentido Giddens (1991) afirma que "la modernidad tardía produce una situación en la que la humanidad deviene bajo ciertos aspectos un solo nosotros que afronta problemas y oportunidades respecto a los cuales no existen otros" (p. 27).

Sin embargo el obstáculo mayor para poder hablar de "identidad global" o de "identidades globales" radica en la dificultad de detectar con precisión un repertorio cultural propiamente global, cuya apropiación subjetiva y distintiva por parte de los actores sociales pudiera dar lugar a un sentimiento de pertenencia también global ad intra (Robertson, 1992). Más aún, como precisa García-Canclini (1999), "la estrategia hegemónica de la globalización suele atender sólo a lo que en estos procesos es reducible al mercado" (p. 50).

Se discrepa de la propuesta de una cultura global porque los argumentos que se esgrimen para defenderla priorizan más las formas objetivadas de cultura: productos, artefactos, información, sobre la in- 
teriorización y auténtica identificación que los sujetos hacen de ellos, que es lo que en esencia significa la identidad, como señala Kapuscinski en un artículo de 2002. Además no se puede prescindir de la riqueza de contenidos y expresiones culturales. Esta riqueza se apoya en dos polos: la inconmensurabilidad de la verdad y la capacidad limitada de los hombres para acceder a ella.

Como apunta García-Canclini (1999):

Al desplazarse el debate sobre la globalizacion en la cuestion de la identidad ... nos negamos a reducirlo a la oposición global/local. Buscamos situarlo en la recomposicion general de lo abstracto y lo concreto en la vida contemporánea, y en la formación de nuevas mediaciones entre ambos extremos. Más que enfrentar identidades esencializadas a la globalización, se trata de indagar si es posible instituir sujetos en estructuras sociales ampliadas (p. 27).

La hipótesis de este artículo es que es posible configurar una identidad multicultural a través de la interiorización de elementos que pertenecen a distintas tradiciones culturales. Esta es la propuesta de Amin Maalouf, quien considera que cuando los elementos culturales que se interiorizan y se asimilan de manera congruente proceden de distintas culturas, la identidad que se configura es una identidad compuesta, ${ }^{2}$ la propia de una persona multicultural.

\section{ESTADO DE LA CUESTIÓN}

Antes de exponer el pensamiento de Maalouf se presentan, de modo sucinto, otras propuestas sobre el tema de la identidad en la era de la globalización.

Después de revisar la bibliografía científica sobre estas proposiciones se considera, que son dos los autores que resultan interesantes por sus aportación sobre la identidad de un individuo que se encuentra vinculado a distintas tradiciones culturales. Los autores elegidos fueron Ulf Hannerz (1992) y Denys Cuche (1996).

2 Expresión acuñada por Maalouf y que se explica más adelante. 


\section{Hannerz: el cosmopolita}

El autor sueco propone la figura del cosmopolita, un sujeto de gran movilidad, abierto al contacto con todas las culturas y que incluso adopta una actitud positiva respecto a la diversidad misma, es decir, respecto a la coexistencia de diferentes culturas en su experiencia personal (Hannerz, 1992).

Sin embargo, como precisa Giménez (2000):

No basta la movilidad para volverse genuinamente cosmopolita. Los migrantes laborales, los turistas internacionales, los exiliados y los expatriados siguen siendo en su mayor parte "localistas de corazón", y por ningún motivo desean desligarse de su lugar de origen (pp. 6-7).

Por lo tanto, Hannerz (1992) sostiene que:

El genuino cosmopolitismo es ante todo una orientación, una voluntad de comprometerse con el otro. Se trata de una actitud intelectual y estética de apertura a las experiencias culturales divergentes, una búsqueda de contrastes más que de uniformidades (p. 239)

Para el autor sueco los cosmopolitas serían los diplomáticos, los hombres de negocios internacionales y ejecutivos transnacionales, además de intelectuales urbanos que se mantienen en contacto a través de redes globales de intercambio cultural.

Frente a la actitud del cosmopolita se contrapone la del localista. "Como tipo ideal, el localista es el que se identifica preferentemente con su cultura local entendida como cultura anclada territorialmente y dinamizada a través de relaciones interpersonales" (Giménez, 2000, p. 6).

La crítica a esta propuesta de Hannerz es la siguiente: desde el punto de vista identitario, difícilmente se puede atribuir al cosmopolita una identidad transcultural y mucho menos global, por que, aunque circula entre diferentes mundos culturales, no llega a ser parte de ninguno de ellos. Según Friedman (1992) el cosmopolita sólo puede desempeñar roles y participar superficialmente en la realidad de otros pueblos, a esto se reduce toda su identidad. 
Si se hace una comparación sintética entre Hannerz y Maalouf se pueden resaltar un punto de unión y otro de diferencia. Comparten la misma premisa de partida: la posibilidad de la coexistencia de diferentes culturas en la experiencia personal. Sin embargo, Hannerz se diferencia de Maalouf en que limita la posibilidad de la identidad cultural múltiple a algunas personas; en cambio el autor de Identidades asesinas afirma que "en todos nosotros coinciden (esas) pertenencias (culturales) múltiples" (Maalouf, 1999, p. 12).

\section{Denys Cuche: la identidad mixta}

Este sociólogo francés presenta algunas reflexiones fundamentales para reformular un nuevo concepto de identidad que se adecue a las características del mundo globalizado.

Cuche (1996) afirma que "cada grupo o individuo, no está encerrado a priori en una identidad unidimensional .... Querer considerar la identidad como monolítica impide comprender los fenómenos de identidad mixta, que son frecuentes en toda sociedad" (p. 91).

El autor precisa que entender la identidad de un modo monolítico deriva en errores como la pretendida doble identidad de jóvenes hijos de inmigrantes, a lo que él contesta diciendo que en estos hijos de inmigrantes no hay dos identidades que se enfrentarían entre ellas y entre las cuales se sentirían rasgados o rotos, sino una única y sincrética (Cuche, 1996).

Así pues, precisa, la dificultad "proviene de la incapacidad de pensar la mezcla cultural" (p. 92). El problema, según Cuche, está en la "cerrazón mental" de algunos para admitir una identidad mixta.

Para el autor es posible que la identidad se configure como una unidad con elementos pertenecientes a otras culturas:

Cada persona configura su identidad a partir de sus diversas pertenencias sociales (de sexo, de edad, de clase social, de grupo cultural, etc.), el individuo que participa de varias culturas elabora a partir de estos diferentes materiales su identidad personal única operando una síntesis original. El resultado es pues una identidad sincrética, y no doble (1996, p. 92).

Y para ilustrar su propuesta menciona varios ejemplos, entre ellos los siguientes: en el Maghreb tradicional no era raro que los miembros de 
antiguas familias judías presentes desde hacía siglos fuesen designados "judíos árabes", dos términos que aparecen poco conciliables desde el auge de los nuevos nacionalismos. En otro contexto, en el Perú contemporáneo existen peruanos calificados como chinos y que se reconocen como tales, descendientes de chinos llegados a Perú en siglo XIX. Se sienten plenamente peruanos, pero permanecen muy atados a su identidad china. Este autor precisa también el caso de Fujimori (Cuche, 1996).

Como se deduce de los textos anteriores, la propuesta del francés no adolece de la limitación que se anotaba en el caso de Hannerz, sino todo lo contrario, ya que presenta un concepto de identidad que reconoce que las personas sean capaces de configurarse partiendo de influencias pertenecientes a distintas culturas.

Las propuestas de Cuche encuentran un reflejo paralelo en las de Maalouf, y no sería extraño que este último las tomara en cuenta, aunque no hace ninguna referencia expresa a este autor.

\section{PROPUESTA DE AMIN MAALOUF}

Maalouf (1999) centra el problema principal al que se enfrentan las personas con distintas influencias culturales en los siguientes términos: "elegir entre afirmar a ultranza su identidad y perderla por completo" (p. 43).

El escritor libanés puntualiza que las dos opciones antes mencionadas tienen su raíz en "esa concepción estrecha, exclusivista, beata y simplista que reduce toda identidad a una sola pertenencia que se proclama con pasión" (1999, p. 13).

La concepción "tribal" de identidad que sigue dominando el mundo entero ... es una concepción heredada de los conflictos del pasado, que muchos rechazaríamos sólo con pensarlo un poco más pero que seguimos suscribiendo por costumbre, por falta de imaginación o resignación, contribuyendo así, sin quererlo, a que se produzcan las tragedias que el día de mañana nos harán sentirnos sinceramente conmovidos (p. 37).

Por lo anterior, y a modo de solución, el autor de Identidades asesinas propone un nuevo modo de entender la identidad personal; un 
concepto de identidad que aúne las distintitas pertenencias culturales que pueden configurar a una persona inmersa en el mundo globalizado.

\section{¿Qué concepto de identidad propone Maalouf?3}

En clave autobiográfica presenta los dos postulados esenciales de su proposición: entender que la identidad está integrada por una pluralidad de elementos culturales y que éstos pueden pertenecer a distintas culturas.

Lo que hace que yo sea yo, y no otro, es ese estar en las lindes de dos países, de dos o tres idiomas, de varias tradiciones culturales. Es eso justamente lo que define mi identidad. ¿Sería acaso más sincero si amputara de mí una parte de lo que soy? (Maalouf, 1999, p. 9).

Esta identidad, identidad compuesta es una identidad con vocación multicultural no obstante Maalouf no utilice este término, por que los elementos culturales que una persona interioriza pertenecen a distintas tradiciones culturales.

Esta identidad compuesta, es una identidad con vocación multicultural no obstante Maalouf...

No está hecha de compartimentos, no se divide en mitades, ni tercios o en zonas estancas. Y no es que tenga varias identidades: tengo solamente una, producto de todos los elementos (culturales) que la han configurado mediante una dosificación singular que nunca es la misma en dos personas (1999, p. 10).

Como afirma el autor libanés, la identidad de una persona no es una yuxtaposición de pertenencias autónomas, no es un mosaico; es un dibujo sobre una piel tirante; basta tocar una sola de esas pertenencias para que vibre la persona entera (1999, p. 34).

A la luz de las anteriores consideración surgen tres preguntas:

3 Su intención no es redefinir el término, se suscribe al concepto de identidad aristotélico en un doble sentido (ontológico y lógico) y lo aplica en un ámbito antropológico social, para referirlo a la persona viviendo en una sociedad mundializada. 


\section{¿Qué configura la identidad compuesta?}

La identidad de una persona está constituida por infinidad de elementos que evidentemente no se limitan a los que figuran en los registros oficiales. La gran mayoría de la gente, desde luego, pertenece a una tradición religiosa; a una nación, y en ocasiones a dos; a un grupo étnico o lingüístico; a una familia más o menos extensa; a una profesión; a una institución; a un determinado ámbito social... Y la lista no acaba ahí sino que prácticamente podría no tener fin (1999, p.18).

Maalouf (1999) especifica que:

No todas esas pertenencias tienen, claro está, la misma importancia, o al menos no la tienen simultáneamente. Pero ninguna de ellas carece por completo de valor. Son elementos constitutivos de la personalidad, casi diríamos de los genes de una alma, siempre que precisemos que en su mayoría no son innatos (p. 19).

A lo anterior hay que añadir que la identidad se va configurando en la trayectoria de vida (tiempo) y tiene como presupuesto la libertad de elección y aceptación.

¿Cómo se descubre la identidad multicultural? La identidad requiere una "autoconciencia", es decir, un conocimiento personal, para lo que él propone un examen de identidad.

Igual que otros hacen examen de conciencia, yo a veces me veo haciendo lo que podríamos llamar "examen de identidad". No trato con ello de encontrar una pertenencia esencial en la que pudiera reconocerme, así que adopto la postura contraria. Rebusco en mi memoria para que aflore el mayor número posible de componentes de mi identidad, los agrupo y hago la lista sin renegar de ninguno de ellos $(1999$, p. 24).

¿Por qué pueden tener una identidad compuesta las personas? Las personas pueden tener una identidad compuesta por "nacimiento o por las vicisitudes de su trayectoria, o incluso porque quieren serlo deliberadamente" (1999, p. 44). 


\section{Reacciones negativas contra la identidad compuesta}

Frente a la persona con identidad compuesta se puede reaccionar de distintas maneras, Maalouf, se detiene a considerar la reacciones negativas, ya que éstas son las que generan identidades asesinas en distintos puntos del mundo.

Rechazo por parte de las personas multiculturales. Las mismas personas pueden rechazar su identidad compuesta; este rechazo puede deberse a dos causas. La primera tiene su origen cuando la identidad compuesta está formada por tradiciones culturales enfrentadas entre sí; “... pertenencias múltiples que a veces se oponen entre sí y nos obligan a elegir, con el consiguiente desgarro" (1999, p. 12).

En todas partes, en todas las sociedades divididas hay un número de hombres y mujeres que llevan en su interior pertenencias contradictorias, que viven en la frontera entre dos comunidades que se enfrentan, seres humanos por los que de algún modo pasan las líneas de fractura étnicas, religiosas o de otro tipo (1999, p. 44).

El autor siendo coherente con su propuesta y proyecto presenta en algunas de sus novelas personajes que tienen una identidad compuesta por tradiciones culturales que se enfrentan, pero que resuelven su identidad personal de un modo integrador. 4

La segunda causa se refiere a la agresión. Maalouf (1999) considera que este factor determina la elección sobre la pertenencia cultural hacia la cual se polariza una persona.

La gente suele tender a reconocerse en la pertenencia que es más atacada; a veces, cuando no se siente con fuerza para defenderla, la disimula y entonces agazapada en la sombra, espera el momento de la revancha; pero, asumida u

4 Un ejemplo es Ossayane Ketabdar protagonista de Las escalas de Levante, este personaje es hijo de un turco y una armenia; su padre le educó en un espíritu abierto: de respeto, convivencia e interés por otras culturas. Participa en el movimiento de resistencia organizado por Charles De Gaulle; en la resistencia conoce y se enamora de una joven de origen judío, con la que se casará y tendrá una hija. 
oculta, proclamada con discreción o con estrépito, es con ella con la que se identifican (1999, p. 34).

Y como consecuencia de ese ataque surge la "'locura asesina', esa propensión de nuestros semejantes a transformarse en asesinos cuando siente que su 'tribu' está amenazada” (1999, p. 36).

Dentro de esta problemática que sufren las personas multiculturales que no aceptan su identidad plural, una mención especial merecen los inmigrantes y sus hijos. Maalouf (1999) refiriéndose a este sector destaca que "es en ese ámbito más que en otros donde las tensiones por causa de la identidad pueden conducir a desviaciones más criminales” (p. 47).

El autor, quien lo ha experimentado en carne propia, presenta las distintas actitudes que se pueden presentar en los inmigrantes. Algunos deciden "no pregonar su diferencia, sino pasar inadvertido. $\mathrm{Su}$ tentación inicial es imitar a sus anfitriones, cosa que algunos consiguen" (1999, p. 46). Otros, ya sea por la frustración de no poder pasar inadvertidos o por decisión propia se muestran, por orgullo, como bravata, más distintos de lo que son. En ambas posturas se advierte que "su frustración desemboca en una contestación brutal” (1999, p. 47).

Rechazo por parte de la sociedad. Como afirma Maalouf (1999) no hay nada en las leyes y en las mentalidades que le permita hoy a la persona multicultural asumir en armonía esa identidad compuesta, y la causa son esos hábitos mentales y esas expresiones que tan arraigadas están en todos nosotros.

El sociólogo presenta tres campos en los cuales se advierte el rechazo de la sociedad al concepto de identidad plural: las leyes, las mentalidades y el lenguaje.

No obstante lo interesante que resulta el enfoque jurídico de las personas con identidad compuesta, el desarrollo del mismo no ha sido abordado por Maalouf y por lo tanto está fuera de los objetivos de esta comunicación; me limitaré a esbozar sus reflexiones en los otros dos ámbitos.

Rechazo en las mentalidades. El autor libanés, como ya se mencionó anteriormente, advierte que hay una "concepción estrecha, exclusivista, beata y simplista que reduce toda identidad a una sola pertenencia que se proclama con pasión" (1999, p. 13). 
Al reflexionar en el porqué de esa mentalidad pienso que la causa principal está en las entrañas mismas del pensamiento moderno, ya que según Ballesteros (2003) la modernidad se puede resumir como exactitud. 5 Y este concepto de exactitud impide que se comprenda la propuesta de Maalouf, por que él define la identidad, no a partir de un único y exacto elemento con el cual se identifica una persona, sino a partir de una variedad de elementos que se integran en una única persona.

\section{Rechazo en el lenguaje}

Por comodidad, englobamos bajo un mismo término a las gentes más distintas, y por comodidad también les atribuimos crímenes, acciones colectivas, opiniones colectivas: "los servios han hecho una matanza...", ... "los negros han incendiado ...", "los árabes se niegan ..." (Maalouf, 1999, p. 29).

Como apunta Rodrigo (1997) no es fácil eliminar los estereotipos negativos que cada cultura tiene de la ajena. A lo largo de la historia los pueblos han deshumanizado a los otros pueblos con el fin de crear un consenso social en contra de ellos. Muchos de estos estereotipos están muy profundamente enraizados en el imaginario colectivo de una cultura, como todavía se puede apreciar en el lenguaje.

A los profesionales de la comunicación les corresponde una labor ardua de implantar, por convencimiento, la costumbre del dialogo intercultural.

\section{Consecuencias del rechazo o ataque a la identidad plural}

La principal consecuencia es aquella que da origen al título del ensayo de Maalouf (1999), generar identidades asesinas: "personas que matarán, que cometerán las perores atrocidades convencidas de que están en su derecho, de que así se ganan el cielo y la admiración de los suyos” (p. 36).

5 El autor detalla que "la modernidad surge en la Florencia de los Medicis, con el descubrimiento de Brunelleschi, en torno a 1420, de la perspectiva ... (la modernidad) va a tener profundas consecuencias en el ámbito del pensamiento general, tratando de desvalorizar progresivamente lo oral a favor de lo visual, lo cualitativo a favor de lo cuantitativo, lo analógico a favor de lo disyuntivo" (Ballesteros, 2003, p. 17). 
Teniendo en cuenta la importancia de este tema, y la clara precisión realista con que el autor lo aborda, considero conveniente presentar la génesis de las matanzas.

Es importante tener como punto de partida que los "autores de matanzas" no lo son a priori.

No creo que la pertenencia a tal o cual etnia, religión, nación u otra cosa predisponga para matar .... Toda comunidad humana, a poco que su existencia se sienta humillada o amenazada, tiende a producir personas que matarán (1999, p. 36).

Así, pues, detonante es la humillación y la amenaza; los que las comparten se sienten solidarios, se agrupan, se movilizan, se dan ánimos entre sí y se aglutinan en en torno a la figura de un líder.

En el seno de cada comunidad herida aparecen evidentemente cabecillas. Airados o calculadores, manejan expresiones extremas que son bálsamo para las heridas. Dicen que no hay que mendigar el respeto de los demás, un respeto que se les debe, sino que hay que imponérselo. Prometen venganza y victoria, inflaman los ánimos .... Pase lo que pase "los otros" se lo habrán merecido y "nosotros" recordaremos con precisión "todo lo que hemos tenido que soportar" desde el comienzo de los tiempos (1999, p. 34).

Y ésta es la "espiral de violencia" con que justifican la agresión que hacen, a cambio de la agresión que reciben o recibieron.

Cuando siente que "los otros" constituyen una amenaza para su etnia (cualquier elemento cultural que los identifique) todo lo que pueden hacer para alejar esa amenaza les parece perfectamente lícito; incluso cuando llegan a la matanza, están convencidos de que se trata de una medida necesaria para preservar la vida de los suyos. Y todos los que les rodean comparten ese convencimiento (1999, p. 39).

Lo más preocupante de esta situación de violencia recíproca radica en la alteración de la conciencia, ya que los autores de las matanzas "suelen tener buena conciencia, y se extrañan de que se les llame cri- 
minales. No pueden serlo, juran, pues ellos sólo tratan de proteger a su ancianas madres, a sus hermanos y hermanas, a sus hijos" (1999, p. 39). “¡Así es como se 'fabrica’ a los autores de matanzas!” (1999, p. 13).

Soluciones de Maalouf ante el problema de la identidad compuesta de las personas multiculturales en un mundo globalizado Maalouf (1999) piensa que el remedio para los conflictos intra e interculturales se encuentra en las personas. Atinadamente centra la solución en la raíz misma del conflicto: en la autoaceptación de la persona con identidad compuesta y multicultural y, por evidente que parezca, afirma que si éstas son "capaces de asumir plenamente su diversidad servirán de 'enlace' entre las diversas comunidades y culturas, y en cierto modo serán el 'aglutinante' de las sociedades en que viven" (1999, p. 44).

Por lo que se refiere a los demás miembros de las comunidades, considera que deben cobrar conciencia sobre lo pernicioso de ciertas frases y adaptar un voluntad de cambio en el uso del lenguaje. Maalouf (1999, p. 30) considera importante que todos cobremos conciencia de que esas frases negativas contra una comunidad cultural, étnica, religiosa, etc., no son inocentes, y de que contribuyen a perpetuar unos prejuicios que han demostrado, a lo largo de toda la historia, su capacidad de perversión y muerte.

En las últimas páginas de su ensayo el escritor libanés recalca la necesidad de concebir la identidad en clave plural y anota un matiz muy importante: que la principal pertenencia que todas las personas comparten es su ser humanos y que por lo tanto todos los hombres forman parte, en último término, de la misma comunidad: la humanidad. Esta pertenencia no anula las diversidades culturales, ya que cada cultura tiene manifestaciones particulares, modos y significados que determinan en cierto modo su ser humanos.

\section{CONCLUSIONES}

El mundo globalizado ofrece un espacio y tiempo de común-vivencia entre distintas comunidades culturales; en muchos casos esta situación de coexistencia implica el riesgo de desencadenar conflictos culturales: 
En todos los lugares en que hoy se viven en vecindad grupos humanos de diferente religión, color, lengua, etnia o nacionalidad; en todos los lugares ... existen tensiones más o menos amortiguadas, más o menos violentas, entre inmigrados y población local, o entre blancos y negros, católicos o protestantes, judíos o árabes, hindúes y sijs, lituanos y rusos, servios y albaneses, griegos y turcos, anglófonos y quebequenses, flamencos y balones, chinos y malayos (Maalouf, 1999, p. 44).

El porqué de ese riesgo está en la realidad de la configuración multicultural de las personas y en la falta de conocimiento y aceptación de esa realidad.

Si esas personas no pueden asumir por sí mismas sus múltiples pertenencias, si se les insta continuamente a que elijan un bando u otro, si se las conmina a reintegrarse en las filas de su tribu (entiéndase cualquier comunidad cultural ya sea de origen étnico, religioso, político, etc.), entonces es lícito que nos inquietemos por el funcionamiento del mundo, (porque se están generando identidades asesinas) (1999, p. 13).

Si nos preguntamos, ¿quién las conmina?, la respuesta es que no sólo los fanáticos y los xenófobos de todas las orillas, también todos nosotros, debido a esos hábitos mentales y a esas expresiones que tan arraigadas están en todos nosotros (Maalouf, 1999).

La autentica solución se encuentra en aceptar una nueva manera de entender la identidad.

Una identidad que se percibirá como la suma de todas nuestras pertenencias, y en cuyo seno la pertenencia a la comunidad humana iría adquiriendo cada vez más importancia hasta convertirse un día en la principal, aunque sin anular por ello todas las demás (1999, p. 109).

Entender a la persona multicultural permitirá una correcta comunicación intercultural y esto será un factor que propicie la paz y el desarrollo en Iberoamérica.

Es necesario que todos sepamos "tejer lazos de unión, disipar malentendidos, hacer entrar en razón a unos, moderar a otros, allanar, re- 
conciliar ... ser puentes, mediadores entre las diversas comunidades y las diversas culturas" (1999, pp. 12-13).

\section{Bibliografia}

Appadurai, A. (2002). Los nuevos territorios de la cultura: globalización, incertidumbre cultural y violencia. En J. Bindé (Ed.), Claves para el siglo XXI. (pp. 181-306). Madrid: Unesco.

Ballesteros, J. (2003). Postmodernidad: decadencia o resistencia. Madrid: Tecnos.

Bauman, Z. (1999). La globlaización: consecuencias humanas. México: FCE.

Bauman, Z. (2001). El enigma multicultural. Un replanteamiento de las identidades nacionales, étinicas y religiosas. Barcelona: Paidós.

Castells, M. (1997). La era de la informacion: economía, sociedad y cultura. Tomo I. Madrid: Alianza.

Cuche, D. (1996). La notion de culture dans les sciencies sociales. París: La Découverte.

Featherstone, M. (Ed.). (1992). Global culture. Londres: Sage.

Friedman, T. (1992) Being the world: globalization and localization. En M. Featherstone (Ed.), Global culture (pp. 311-328). Londres: Sage.

García-Canclini, N. (1999). La globalización imaginada. Buenos Aires: Paidós.

Giddens, A. (1991). Sociología. Madrid: Alianza.

Giddens, A. (2000). Un mundo desbocado. Los efectos de la globalización en nuestras vidas. Madrid: Taurus.

Giménez, G. (2000). Identidades en globalización. Espiral, 7, (19), 27-48.

Giménez, G. (2005). Cultura, identidad y metropolitanismo globla. Revista Mexicana de Sociología, 67 (3); 483-512,

Hannerz, U. (1992). Cosmopolitan and locals in world culture. En M. Featherstone (Ed.), Global culture (pp. 237-251). Londres: Sage.

Kapuscinski, R. (2002). Globalización y diversificación. Claves de la razón práctica, 126, 20-25.

Maalouf, A. (1988). León el Africano. Madrid: Alianza.

Maalouf, A. (1989). Samarcanda. Madrid: Alianza.

Maalouf, A. (1991). Los jardínes de la luz. Madrid: Alianza.

Maalouf, A. (1993). La roca de Tanios. Madrid: Alianza.

Maalouf, A. (1996). Las cruzads vistas por los árabes. Madrid: Alianza. 
Maalouf, A. (1997). Las escalas de Levante. Madrid: Alianza.

Maalouf, A. (1999). Identidades asesinas. Madrid: Alianza.

Maalouf, A. (2001). El viaje de Baldassare. Madrid: Alianza.

Maalouf, A. (2002). El amor de lejos. Madrid: Alianza.

Roberston, R. (1992). Mapping the global condition: globalisation as the central concept. En M. Featherstone (Ed.), Global culture (pp. 15-30). Londres: Sage.

Rodrigo, M. (1997). Elementos para una comunicación intercultural. Revista CIDOB d 'Afers Internacionals, 36, 12-20.

Toscano, R. (2001). Identidad de grupo. Diversidad y conviviencia entre ética y políticas. Claves de la razón práctica, 114, 22-31. 